\title{
Thermochemistry of heteroatomic compounds: Analysis of thermodynamic functions of Mendeleev's Periodic table atoms in various phases; possibility for the bond strengths calculations in condensed state
}

\author{
Vitaly Vitalevich Ovchinnikov \\ Department of General Chemistry and Ecology, Kazan National Research Technical University Named after A. N. Tupolev, St-K. Marks 10, \\ Tatarstan, Russia
}

\section{Email address: \\ chem_vvo@mail.ru}

\section{To cite this article:}

Vitaly Vitalevich Ovchinnikov. Thermochemistry of Heteroatomic Compounds: Analysis of Thermodynamic Functions of Mendeleev's Periodic Table Atoms in Various Phases; Possibility for the Bond Strengths Calculations in Condensed State. American Journal of Physical Chemistry. Vol. 3, No. 5, 2014, pp. 54-60. doi: 10.11648/j.ajpc.20140305.11

\begin{abstract}
The values of free energies, the heats and entropies of formation and heat capacities of gaseous and condensed atoms of I-VII main and lateral groups and three groups of transitive elements of Mendeleev's Periodic table were analyzed. Seventy nine equations of such type as $\Delta_{\mathrm{g}, \mathrm{f}, \mathrm{s}} \Psi^{\circ}=i \pm f N$, in which $\Delta_{\mathrm{g}, \mathrm{f}, \mathrm{s}} \Psi^{\rho}$ is thermodynamic function, $i$ and $f$ are stoichiometric coefficients, have been calculated. The obtained equations can be used for the calculations of the same functions for other atoms, for with they are not known. The same equations can be used for the next calculation of bond strength in they also. Instead of bond energy $\left(E_{\mathrm{b}}\right)$ or bond dissociation energy (BDE) the bond strength $\left(S_{\mathrm{b}}\right)$ new symbol of the bond powers for organic and organometallic substances has been suggested. It has been done because there are all thermodynamic functions for practically all atoms of Periodic table and hard to define the bond power with the use the heat function (enthalpy) only; probably is necessary to present the all thermodynamic parameters for the calculating bonds between atoms.
\end{abstract}

Keywords: Atom, Free Energy, Heat of Formation, Entropy, Heat of Capacity, Bond Energy, Strength of Bond

\section{Introduction}

The bond energies of organic and bioorganic compounds define not only a direction and energy of organic reactions, but the possibility and activity of the live organisms also. Their values and the importance draw attention of the researchers in chemistry and biochemistry during very big period of time $[1,2]$.

It is known, that the new formation and destruction of the old bonds in organic and biochemical molecules play a very important significance for the understanding of synthetic ways of the mentioned substances not only for an industry, but for the vital objects. The important attention to this process is shown not only synthetic, but the theoretical biochemists also.

\section{Results and Discussion}

The quantitative definition of bond energy is connected both with labour-consuming physical experiment and with the different theoretical calculations. One of such calculations is based on the data about the heat of atomization $\left(\Delta_{\mathrm{a}} H^{\circ}\right)$ an organic molecule in a gas phase. In turn, the heat of atomization depends on the sum of the heats of its atoms $\left(\Delta_{\mathrm{at}} H_{\text {gas }}^{\mathrm{o}}\right)$ and the heat (enthalpy) of molecule formation in a gas phase $\left(\Delta_{\mathrm{f}} H_{\text {gas }}^{\mathrm{o}}\right)[1,2]$. Necessary to note, that the heat of atomization is equal to the sum of all bond energies of $\left(E_{\mathrm{b}}\right)$ in such molecule also (Eq. 1).

$$
\Delta_{\mathrm{a}} H^{\mathrm{o}}=\Sigma \Delta_{\mathrm{at}} H_{\text {gas }}^{\mathrm{o}}-\Delta_{\mathrm{f}} H_{\text {gas }}^{\mathrm{o}}=\Sigma E_{\mathrm{b}}
$$

Earlier we conducted the analysis of thermodynamic functions $\left(\Delta_{\mathrm{c}, \mathrm{f}, \mathrm{s}} \Psi^{\circ}\right)$ of free energies $\left(\Delta_{\mathrm{c}, \mathrm{f}} G^{0}\right)$, the heats of combustion and formation $\left(\Delta_{\mathrm{c}, \mathrm{f}} H^{\circ}\right)$ and formation entropy $\left(S^{\circ}\right)$ in condensed and gaseous phases of organic compounds IVII of groups of Mendeleev's Periodic system of elements. Also it has been found that all functions depend from the number of valence electrons $N$ without of the number $(h)$ of lone electron pairs $(g)$ of all investigated molecules (Eq. 2) 


$$
\Delta_{\mathrm{g}, \mathrm{f}, \mathrm{s}} \Psi^{\circ}=i \pm f^{*}(N-h g)
$$

here $i$ and $f$ are stoichiometric coefficients

Also we have become interested not only the heat of formation of simple atoms in gaseous and condensed states, but and their other thermodynamic functions, which are known in the literature and summed in Table $1[8,9]$.

After the painstaking analysis of all thermodynamic functions and the heat of capacities of gaseous and condensed atoms was found that all they depend on the general number of electrons $N$ in atoms I-VIII of groups in the different periods only (Table 1 and equations in it). Therefore the equation (2) necessary was changed into equation (3) before the use

$$
\Delta_{\mathrm{g}, \mathrm{f}, \mathrm{S}} \Psi^{\circ}=i \pm f^{*} N
$$

The correlations like (3) established and presented in Table 1 allow to calculate the unknown values of thermodynamic functions in the condensed condition for such necessary for the formation of organic molecules atoms as hydrogen, calcium, oxygen and others.

However there are some positions in Table 1 in the last column, in which there are not real correlations; they are marked as "no correlations".

The above mentioned correlations are necessary for the subsequent calculations of thermodynamic parameters of such important heteroatomic compounds as sugars, amino acids and others useful in the industry, biochemistry and medicine of substances. These data are collected in Table 2 and presented in square cells.

Nevertheless at the consideration of the data in Table 1 there are some questions and offers for their discussion. One of them is connected with the use of the thermodynamic characteristic for the name of the bond energy. Till now for the designation of bond energy $\left(E_{\mathrm{b}}\right)$ or bond dissociation energy (BDE) was used the heat function (enthalpy) only [2].

It is known, that there are practically all thermodynamic functions $\left(\Delta_{\mathrm{f}} G^{\mathrm{o}}, \Delta_{\mathrm{f}} H^{\mathrm{o}}, \mathrm{f}_{\mathrm{f}}^{\mathrm{o}}\right.$ and $C_{\mathrm{p}}$, see Table 1 also) for simple atoms in the literature, which can be used for the calculations and description of any bonds in gaseous and condensed phases. That's why we suggest to use for all heteroatomic bonds by the term "strength of bond $\left(S_{\mathrm{b}}\right)$ " but not "bond energy or bond dissociation energy" in organic molecule and to show all thermodynamic functions of examined bond in molecules.

For the revision of this offer we have chosen a some linear and cyclic alkanes and have defined for them the basic thermodynamic functions and their sizes of atomizations in gas and condensed phases: $\left(\Delta_{\mathrm{g}, \mathrm{f,s}} \Psi^{\circ}\right.$, Table 3$)$.

The analysis of the results of Table 3 shows that all atomizations of all chosen thermodynamic functions (values of heat capacities were not considered) of different alkanes are depended from the number of valence electrons (Eqs. 4-8; there is bad correlation between $\Delta_{\mathrm{a}} G_{\text {cond }}^{\mathrm{o}}$ and $\left.N, r 0.763\right)$, as it was shown earlier for the organic molecules [3-7 ].

The C-H and C-C bond strengths $( \pm 1.5 \%)$ of alkanes can calculated with the use of Eq. 1, but instead the enthalpy could be used the other functions - free energy and entropy (Table 4).

$$
\begin{aligned}
& \Delta_{\mathrm{a}} G_{\text {gas }}^{\mathrm{o}}=(-20.6 \pm 48.0)+(145.1 \pm 1.6) N, r 0.999, S_{0} 55.6, n \\
& 7 \text {, compounds (I-VII) } \\
& \Delta_{\mathrm{a}} H_{\text {gas }}^{\mathrm{o}}=(90.5 \pm 46.6)+(194.2 \pm 1.5) N, r 0.999, S_{\mathrm{o}} 54.0, n 7 \text {, } \\
& \text { compounds (I-VII) }
\end{aligned}
$$

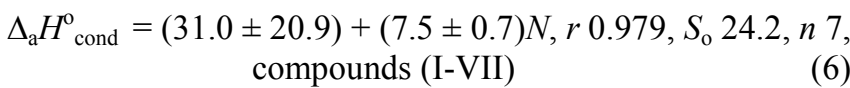

$$
\begin{aligned}
& { }_{\mathrm{a}} S_{\text {gas }}^{\mathrm{o}}=(-1.7 \pm 37.0)+(67.1 \pm 1.2) N, r 0.999, S_{\mathrm{o}} 49.9, n 7 \text {, } \\
& \text { compounds (I-VII) } \\
& { }_{a} S^{\mathrm{o}}{ }_{\text {cond }}=(9.9 \pm 49.8)+(7.8 \pm 1.6) N, r 0.903, S_{0} 57.6, n 7 \text {, } \\
& \text { compounds (I-VII) }
\end{aligned}
$$

\begin{tabular}{|c|c|c|c|c|c|c|c|}
\hline \multicolumn{8}{|c|}{ First Main Group } \\
\hline Functions, $\Psi^{\circ}$ & Atoms, $N /$ Phases & $\mathrm{Li}, 3$ & $\mathrm{Na}, 11$ & K, 19 & $\mathrm{Rb}, 37$ & Cs, 55 & Equations \\
\hline \multirow{2}{*}{$\Delta_{\mathrm{f}} G^{\mathrm{o}}$} & gaseous & 128.0 & 77.8 & 61.2 & 54.0 & 50.9 & $\begin{array}{l}\Delta_{\mathrm{f}} G^{\mathrm{o}}=(104.2 \pm 16.8)-(1.2 \pm 0.5) N r 0.787, \\
S_{o} 22.5, n 5^{\mathrm{a}}\end{array}$ \\
\hline & condense & 0.9 & 0.5 & 0.3 & & & $\begin{array}{l}\Delta_{\mathrm{f}} G^{\mathrm{o}}=(1.0 \pm 0.1)-(0.04 \pm 0.01) N r 0.981 \\
S_{o} 0.1, n 3^{\mathrm{a}}\end{array}$ \\
\hline \multirow{2}{*}{$\Delta_{\mathrm{f}} H^{\mathrm{o}}$} & gaseous & 105.5 & 107.3 & 89.0 & 80.9 & 76.5 & $\begin{array}{l}\Delta_{\mathrm{S}} H^{\circ}=(107.3 \pm 7.7)-(0.6 \pm 0.2) N r 0.963 \\
S_{o} 0.04, n 5^{\mathrm{b}}\end{array}$ \\
\hline & condensed & 2.4 & 2.4 & 2.3 & 2.2 & 2.1 & $\begin{array}{l}\Delta_{\mathrm{H}} H^{\mathrm{o}}=(2.4 \pm 0.03)-(0.02 \pm 0.01) N r 0.987, \\
S_{o} 0.02, n 5^{\mathrm{b}}\end{array}$ \\
\hline \multirow{2}{*}{${ }_{\mathrm{f}} S^{\mathrm{O}}$} & gaseous & 138.7 & 153.6 & 160.3 & 170.1 & 175.6 & $\begin{array}{l}{ }^{\circ} S^{0}=(143.4 \pm 4.0)+(0.7 \pm 0.1) N r 0.942, S_{o} \\
5.6, n 5^{\mathrm{b}}\end{array}$ \\
\hline & condensed & 29.1 & 51.5 & 64.6 & 76.8 & 85.1 & $\begin{array}{l}S^{o}=(36.9 \pm 6.9)+(1.0 \pm 0.2) N r 0.932, S_{o} \\
9.3, n 5^{b}\end{array}$ \\
\hline $\begin{array}{l}C p \\
\text { First Lateral G }\end{array}$ & condensed & 31.3 & 32.7 & 32.7 & 31.4 & 31.4 & No correlation \\
\hline
\end{tabular}

The data of Table 4 shows that the bond strength of all chosen gaseous functions are on about two order more than for condensed phase. Probably it can explain that alkanes in the condensed phase have a sensitive dispersion interaction between $\mathrm{C}-\mathrm{H}$ bonds each other. At the same time to hard explain the some negative values of $\mathrm{C}-\mathrm{C}$ bond strength for linear and cyclic alkanes (!).

Table 1. Thermodynamic functions $\left(\mathrm{kJ} \mathrm{mol}^{-1}, \mathrm{~J} \mathrm{~mol}^{-1} \mathrm{~K}^{-1}\right)$ of atoms in the gaseous and condensed states of different groups of Periodic Table 


\begin{tabular}{|c|c|c|c|c|c|c|c|}
\hline Functions, $\Psi^{\circ}$ & Atoms, $N /$ Phases & & $\mathrm{Cu}, 29$ & $\mathrm{Ag}, 47$ & $\mathrm{Au}, 79$ & & Equations \\
\hline$\Delta_{\mathrm{f}} G^{\mathrm{o}}$ & gaseous & & 299.7 & 247.4 & 325.6 & & No correlation \\
\hline \multirow[t]{2}{*}{$\Delta_{\mathrm{f}} H^{\mathrm{o}}$} & gaseous & & 337.6 & 284.9 & 83.7 & & $\begin{array}{l}\Delta_{\mathrm{f}} H^{\mathrm{o}}=(505.0 \pm 48.5)-(5.2 \pm 0.9) N r 0.986, \\
S_{o} 31.2, n 3^{\mathrm{b}}\end{array}$ \\
\hline & condensed & & 11.9 & & & & \\
\hline \multirow[t]{2}{*}{${ }_{5} S^{\circ}$} & gaseous & & 166.4 & 172.0 & 180.4 & & $\begin{array}{l}{ }_{f} S^{0}=(158.6 \pm 0.8)+(0.3 \pm 0.01) N r 0.998, \\
S_{o} 0.5, n 3^{\mathrm{b}}\end{array}$ \\
\hline & condensed & & 33.3 & 42.6 & & & \\
\hline$C p$ & condensed & & 24.5 & 25.5 & & & \\
\hline \multicolumn{8}{|c|}{ Second Main Group } \\
\hline Functions, $\Psi^{\circ}$ & Atoms, $N /$ Phases & $\mathrm{Be}, 4$ & $\mathrm{Mg}, 12$ & $\mathrm{Ca}, 20$ & $\mathrm{Sr}, 38$ & Ba, 56 & Equations \\
\hline \multirow{2}{*}{$\Delta_{\mathrm{f}} G^{\mathrm{o}}$} & gaseous & 288.1 & 114.4 & 142.8 & 130.1 & 143.2 & $\begin{array}{l}\Delta_{\mathrm{f}} G^{\mathrm{o}}=(106.2 \pm 1.3)+(0.6 \pm 0.02) \mathrm{Nr} \\
0.999, S_{o} 1.1, n 3(\text { no } \mathrm{Mg}, \mathrm{Sr})^{\mathrm{a}}\end{array}$ \\
\hline & condensed & 10.0 & 6.1 & & & 136.1 & $\begin{array}{l}\Delta_{\mathrm{f}} G^{\mathrm{o}}=(-11.7 \pm 14.8)+(2.6 \pm 0.4) N r 0.986 \\
S_{o} 17.7, n 3\left(\text { no Sr,Ca) }{ }^{\mathrm{a}}\right.\end{array}$ \\
\hline \multirow[t]{2}{*}{$\Delta_{\mathrm{f}} H^{\mathrm{o}}$} & gaseous & 327.4 & 149.0 & 176.6 & 163.5 & 174.6 & $\begin{array}{l}\Delta_{\mathrm{f}} H^{\mathrm{o}}=(320.4 \pm 62.2)-(4.7 \pm 2.5) N r 0.884, \\
S_{o} 60.1, n 3(\mathrm{no} \mathrm{Mg}, \mathrm{Ba})^{\mathrm{a}}\end{array}$ \\
\hline & condensed & 12.0 & 9.0 & 7.79 & 0.91 & 4.99 & No correlation \\
\hline \multirow{2}{*}{${ }_{f} S^{\circ}$} & gaseous & 136.1 & 148.5 & 154.8 & 164.5 & 170.1 & $\begin{array}{l}S^{\mathrm{o}}=(138.9 \pm 3.4)+(0.6 \pm 0.1) N r 0.956, S_{o} \\
4.5, n 5^{\mathrm{a}}\end{array}$ \\
\hline & condensed & 9.4 & 32.7 & 45.5 & 50.9 & 62.5 & $\begin{array}{l}{ }_{i} S^{0}=(17.3 \pm 7.4)+(0.9 \pm 0.2) N r 0.910, S_{o} \\
9.7, n 5^{\mathrm{b}}\end{array}$ \\
\hline$C p$ & condensed & 15.9 & 24.9 & 26.3 & 27.3 & 28.3 & $\begin{array}{l}C p=(24.4 \pm 0.4)+(0.07 \pm 0.01) N r 0.970, \\
S_{o} 0.4, n 4(\text { no Be })^{\mathrm{a}}\end{array}$ \\
\hline \multicolumn{8}{|c|}{ Second Lateral Group } \\
\hline Functions, $\Psi^{\circ}$ & Atoms, $N /$ Phases & & $\mathrm{Zn}, 30$ & $\mathrm{Cd}, 48$ & $\mathrm{Hg}, 80$ & & Equations \\
\hline$\Delta_{\mathrm{f}} G^{\mathrm{o}}$ & gaseous & & 94.9 & 77.4 & 31.8 & & $\begin{array}{l}\Delta_{\mathrm{f}} G^{\mathrm{o}}=(135.5 \pm 6.6)-(1.3 \pm 0.1) N r 0.995, \\
S_{o} 4.2, n 3^{\mathrm{a}}\end{array}$ \\
\hline \multirow[t]{2}{*}{$\Delta_{\mathrm{f}} H^{\mathrm{o}}$} & gaseous & & 128.6 & 111.9 & 61.3 & & $\begin{array}{l}\Delta_{\mathrm{f}} H^{\mathrm{o}}=(172.9 \pm 9.5)-(1.4 \pm 0.1) N r 0.992, \\
S_{o} 6.9, n 3^{\mathrm{a}}\end{array}$ \\
\hline & $\begin{array}{l}\text { condensed } \\
\text { gaseous }\end{array}$ & & 6.52 & 167.6 & 174.8 & & \\
\hline${ }_{\mathrm{H}} \mathrm{S}^{\mathrm{o}}$ & condensed & & 41.6 & 51.7 & 76.0 & & $\begin{array}{l}{ }_{\mathrm{f}} S^{\mathrm{o}}=(19.8 \pm 2.9)+(0.7 \pm 0.05) N r 0.997, S_{o} \\
1.8, n 3^{\mathrm{a}}\end{array}$ \\
\hline$C p$ & condensed & & 25.3 & 26.0 & 28.0 & & $\begin{array}{l}C p=(23.5 \pm 0.3)+(0.05 \pm 0.01) N r 0.993, \\
S_{o} 0.2, n 3^{\text {a }}\end{array}$ \\
\hline \multicolumn{8}{|c|}{ Third Main Group } \\
\hline Functions, $\Psi^{\circ}$ & Atoms, $N /$ Phases & $\mathrm{B}, 5$ & $\mathrm{Al}, 13$ & $\mathrm{Ga}, 31$ & In, 39 & $\mathrm{~T} 1,81$ & Equations \\
\hline \multirow[t]{2}{*}{$\Delta_{\mathrm{f}} G^{\mathrm{o}}$} & gaseous & 545.2 & 285.8 & 233.8 & 208.1 & 145.1 & $\begin{array}{l}\Delta_{\mathrm{f}} G^{\mathrm{o}}=(299.8 \pm 13.6)-(2.0 \pm 0.3) N r 0.970, \\
S_{o} 18.4, n 4(\text { no B })^{\mathrm{a}}\end{array}$ \\
\hline & condensed & 19.3 & 7.2 & & & & \\
\hline \multirow{2}{*}{$\Delta_{\mathrm{f}} H^{\mathrm{o}}$} & gaseous & 556.5 & 326.3 & 272.0 & 238.5 & 179.9 & $\begin{array}{l}\Delta_{\mathrm{f}} H^{\mathrm{o}}=(338.8 \pm 17.7)-(2.1 \pm 0.4) N r 0.975, \\
S_{o} 16.7, n 4(\text { no B })^{\mathrm{a}}\end{array}$ \\
\hline & condensed & 22.0 & 10.0 & 246.8 & & & $\begin{array}{l}\Delta_{\mathrm{f}} H^{\mathrm{o}}=(-60.7 \pm 67.4)+(9.4 \pm 3.9) N r 0.939, \\
S_{o} 64.7, n 3^{\mathrm{a}}\end{array}$ \\
\hline \multirow{3}{*}{$\mathrm{f}^{\circ}$} & gaseous & 153.3 & 164.4 & & & & \\
\hline & condensed & 14.8 & 37.8 & 40.9 & 57.8 & 64.2 & $\begin{array}{l}\mathrm{S} S^{\circ}=(23.9 \pm 7.9)+(0.6 \pm 0.2) N r 0.873, S_{o} \\
10.8, n 5^{\text {a }}\end{array}$ \\
\hline & gaseous & & 21.4 & 25.3 & & & \\
\hline$C p$ & condensed & & 29.3 & 26.9 & 26.7 & 26.3 & $\begin{array}{l}C p=(28.8 \pm 1.0)+(0.04 \pm 0.02) N r 0.775 \\
S_{o} 1.0, n 4^{\text {a }}\end{array}$ \\
\hline \multicolumn{8}{|c|}{ Third Lateral Group } \\
\hline Functions, $\Psi^{\circ}$ & Atoms, $N /$ Phases & & Sc, 21 & $\mathrm{Y}, 39$ & La, 57 & & Equations \\
\hline \multirow[t]{2}{*}{$\Delta_{\mathrm{f}} G^{\mathrm{o}}$} & gaseous & & 302.2 & 387.0 & 381.8 & & $\begin{array}{l}\Delta_{\mathrm{f}} G^{\mathrm{o}}=(270.8 \pm 60.1)+(2.2 \pm 1.4) N r \\
0.837, S_{o} 36.7, n 3^{\mathrm{a}}\end{array}$ \\
\hline & condensed & & & & 430.8 & & \\
\hline \multirow[t]{2}{*}{$\Delta_{\mathrm{f}} H^{\mathrm{o}}$} & gaseous & & 381.6 & 426.8 & 430.9 & & $\begin{array}{l}\Delta_{\mathrm{f}} H^{0}=(359.2 \pm 30.1)+(1.2 \pm 0.7) \mathrm{Nr} \\
0.901, S_{o} 16.8, n 3^{\mathrm{a}}\end{array}$ \\
\hline & condense & & & & 431.8 & & \\
\hline \multirow{3}{*}{${ }_{\mathrm{S}} \mathrm{S}^{\mathrm{O}}$} & gaseous & & $1 / 4.1$ & & 182.3 & & ${ }_{\oplus} S^{0}=(25.8 \pm 1.8)+(0.5 \pm 0.04) N r 0.997, S_{o}$ \\
\hline & condensed & & 37.6 & 46.0 & 57.1 & & $1.1, n 3^{\mathrm{a}}$ \\
\hline & gaseous & & 22.1 & & 22.8 & & \\
\hline$C p$ & condensed & & 25.1 & 25.1 & 27.2 & & $\begin{array}{l}C p=(23.5 \pm 1.4)+(0.06 \pm 0.03) N r 0.866 \\
S_{o} 0.8, n 3^{\text {a }}\end{array}$ \\
\hline \multicolumn{8}{|c|}{ Fourth Main Group } \\
\hline Functions, $\Psi^{\circ}$ & Atoms, $N /$ Phases & C, 6 & $\mathrm{Si}, 14$ & Ge, 32 & Sn, 50 & $\mathrm{~Pb}, 82$ & Equations \\
\hline$\Delta_{\mathrm{f}} G^{\mathrm{o}}$ & gaseous & 669.5 & 399.1 & 335.8 & 267.3 & 162.9 & $\Delta_{\mathrm{f}} G^{\mathrm{o}}=(569.7 \pm 78.1)-(5.5 \pm 1.7) N r 0.881$, \\
\hline
\end{tabular}




\begin{tabular}{|c|c|c|c|c|c|c|c|}
\hline \multirow{4}{*}{$\Delta_{\mathrm{f}} H^{\mathrm{o}}$} & & & & & & & $S_{o} 104.0, n 5^{\mathrm{a}}$ \\
\hline & condensed & 2.9 & & & & 2.2 & \\
\hline & gaseous & 715.0 & 468.7 & 376.6 & 302.1 & 195.8 & $\begin{array}{l}\Delta_{\mathrm{f}} H^{\mathrm{o}}=(626.7 \pm 73.1)-(5.8 \pm 1.6) N r 0.904, \\
S_{o} 97.3, n 5^{\mathrm{a}}\end{array}$ \\
\hline & condensed & 16.5 & 50.6 & & 2.1 & 4.3 & No correlation \\
\hline \multirow{2}{*}{${ }_{f} S^{\circ}$} & gaseous & 157.9 & 167.9 & 167.8 & & 175.3 & $\begin{array}{l}S^{\mathrm{o}}=(158.3 \pm 2.9)+(0.2 \pm 0.01) N r 0.966, S_{\mathrm{o}} \\
3.2, n 3(\text { no Si,Sn })^{\mathrm{a}}\end{array}$ \\
\hline & condensed & 5.8 & 47.2 & 31.1 & 51.4 & 71.7 & $\begin{array}{l}S^{\mathrm{o}}=(16.5 \pm 11.6)+(0.7 \pm 0.2) N r 0.839, S_{\mathrm{o}} \\
15.5, n 5^{\mathrm{a}}\end{array}$ \\
\hline \multirow[t]{2}{*}{$C p$} & gaseous & 20.4 & 22.2 & 30.7 & & & $\begin{array}{l}C p=(17.3 \pm 1.2)+(0.4 \pm 0.06) N r 0.990, S_{o} \\
1.1, n 3^{\mathrm{a}}\end{array}$ \\
\hline & condensed & 6.1 & 25.6 & 23.3 & 27.0 & 26.8 & No correlation \\
\hline \multicolumn{8}{|c|}{ Fourth Lateral Group } \\
\hline Functions, $\Psi^{\circ}$ & Atoms, $N /$ Phases & & $\mathrm{Ti}, 22$ & $\mathrm{Zr}, 40$ & Hf, 72 & & Equations \\
\hline \multirow[t]{2}{*}{$\Delta_{\mathrm{f}} G^{\mathrm{o}}$} & gaseous & & 429.9 & 568.4 & 660.8 & & $\begin{array}{l}\Delta_{\mathrm{f}} G^{\mathrm{o}}=(355.8 \pm 61.3)+(4.4 \pm 1.2) N r 0962, \\
S_{o} 44.6, n 3^{\mathrm{a}}\end{array}$ \\
\hline & condensed & & 14.8 & 18.7 & & & \\
\hline \multirow[t]{2}{*}{$\Delta_{\mathrm{f}} H^{\circ}$} & gaseous & & 474.5 & 610.9 & 702.9 & & $\begin{array}{l}\Delta_{\mathrm{H}} H^{\mathrm{o}}=(400.9 \pm 60.0)+(4.3 \pm 1.2) \mathrm{Nr} \\
0.963, S_{o} 43.7, n 3^{\mathrm{a}}\end{array}$ \\
\hline & condensed & & 16.2 & 22.0 & & & \\
\hline \multirow{2}{*}{${ }_{f} S^{\circ}$} & gaseous & & 180.3 & 183.0 & 186.9 & & $\begin{array}{l}{ }^{\mathrm{o}}=(177.6 \pm 0.4)+(0.1 \pm 0.02) N r 0.998, S_{\mathrm{o}} \\
0.3, n 3^{\mathrm{b}}\end{array}$ \\
\hline & condensed & & 39.18 & 47.60 & 71.94 & & $\begin{array}{l}\mathrm{f}^{\mathrm{o}}=(23.1 \pm 3.7)+(0.7 \pm 0.1) N r 0.993, S_{\mathrm{o}} \\
2.7, n 3^{\mathrm{b}}\end{array}$ \\
\hline \multirow[b]{2}{*}{$C p$} & gaseous & & 24.4 & 26.6 & & & \\
\hline & condensed & & 33.5 & 25.7 & 25.5 & & $\begin{array}{l}C p=(34.6 \pm 5.4)-(0.1 \pm 0.1) N r 0.789, S_{o} \\
4.0, n 3^{\mathrm{a}}\end{array}$ \\
\hline \multicolumn{8}{|c|}{ Fifth Main Group } \\
\hline Functions, $\Psi^{\circ}$ & Atoms, $N /$ Phases & $\mathrm{N}, 7$ & $\mathrm{P}, 15$ & As, 33 & $\mathrm{Sb}, 51$ & $\mathrm{Bi}, 83$ & Equations \\
\hline \multirow[t]{2}{*}{$\Delta_{\mathrm{r}} G^{\mathrm{o}}$} & gaseous & 455.6 & 292.1 & 247.3 & 222.2 & 168.2 & $\begin{array}{l}\Delta_{\mathrm{f}} G^{\mathrm{o}}=(313.1 \pm 6.9)-(1.8 \pm 0.1) N r 0.994, \\
S_{o} 6.7, n 4(\text { no N })^{\mathrm{a}}\end{array}$ \\
\hline & condensed & & 12.1 & & & & \\
\hline \multirow{2}{*}{$\Delta_{\mathrm{f}} H^{\circ}$} & gaseous & 472.8 & 333.9 & 288.7 & 262.3 & 207.1 & $\begin{array}{l}\Delta_{\mathrm{f}} H^{\mathrm{o}}=(355.7 \pm 6.7)-(1.8 \pm 0.1) N r 0.994, \\
S_{o} 6.5, n 4\left(\text { no N) }{ }^{\mathrm{a}}\right.\end{array}$ \\
\hline & condensed & 316.4 & 18.1 & 13.5 & 10.6 & & $\begin{array}{l}\Delta_{\mathrm{f}} H^{\mathrm{o}}=(20.9 \pm 10.0)-(0.2 \pm 0.02) N r 0.991, \\
S_{o} 0.7, n 3(\mathrm{P}, \mathrm{As}, \mathrm{Sb})^{\mathrm{a}}\end{array}$ \\
\hline \multirow{2}{*}{${ }_{f} S^{0}$} & gaseous & 153.2 & 163.1 & 174.1 & & 186.9 & $\begin{array}{l}S^{\mathrm{o}}=(155.4 \pm 4.2)+(0.4 \pm 0.1) N r 0.950, S_{\mathrm{o}} \\
5.5, n 4(\text { no Sb })^{\mathrm{a}}\end{array}$ \\
\hline & condensed & & 41.1 & 35.1 & 45.7 & 56.8 & $\begin{array}{l}\mathrm{f}^{\mathrm{o}}=(38.4 \pm 4.4)+(0.2 \pm 0.07) N r 0.933, S_{\mathrm{o}} \\
3.7, n 3(\text { no As })^{\mathrm{a}}\end{array}$ \\
\hline \multirow{2}{*}{$C p$} & gaseous & 32.9 & 20.8 & 20.8 & & 20.8 & No correlation \\
\hline & condensed & & 26.3 & 24.7 & 25.2 & 25.6 & No correlation \\
\hline \multicolumn{8}{|c|}{ Fifth Lateral Group } \\
\hline Functions, $\Psi^{\circ}$ & Atoms, $N /$ Phases & & $\mathrm{V}, 23$ & $\mathrm{Nb}, 41$ & $\mathrm{Ta}, 73$ & & Equations \\
\hline$\Delta_{\mathrm{f}} G^{\mathrm{o}}$ & gaseous & & 468.0 & 698.0 & 738.7 & & $\begin{array}{l}\Delta_{\mathrm{f}} G^{\mathrm{o}}=(409.7 \pm 149.7)+(4.9 \pm 3.0) N r \\
0.855, S_{o} 106.8, n 3^{\mathrm{a}}\end{array}$ \\
\hline \multirow[t]{2}{*}{$\Delta_{\mathrm{f}} H^{\circ}$} & gaseous & & 515.3 & 721.9 & 781.6 & & $\begin{array}{l}\Delta_{\mathrm{f}} H^{\mathrm{o}}=(448.4 \pm 124.9)+(4.9 \pm 2.0) \mathrm{Nr} \\
0.892, S_{o} 89.2, n 3^{\mathrm{a}}\end{array}$ \\
\hline & condensed & & 17.3 & 29.6 & & & \\
\hline \multirow{3}{*}{${ }_{\mathrm{f}} S^{\mathrm{o}}$} & gaseous & & 180.3 & 183.0 & 186.9 & & $\begin{array}{l}S^{\mathrm{o}}=(177.4 \pm 0.4)+(0.1 \pm 0.02) N r 0.998, S_{\mathrm{o}} \\
0.3, n 3^{\mathrm{b}}\end{array}$ \\
\hline & condensed & & 28.7 & 36.5 & 41.5 & & $\begin{array}{l}S^{\mathrm{o}}=(24.4 \pm 3.6)+(0.2 \pm 0.07) N r 0.959, S_{\mathrm{o}} \\
2.6, n 3^{\mathrm{a}}\end{array}$ \\
\hline & gaseous & & 24.5 & & 20.9 & & \\
\hline$C p$ & condensed & & 24.2 & 24.9 & 25.3 & & $\begin{array}{l}C p=(23.8 \pm 0.3)+(0.02 \pm 0.01) N r 0.950, \\
S_{o} 0.2, n 3^{\text {a }}\end{array}$ \\
\hline \multicolumn{8}{|c|}{ Sixth Main Group } \\
\hline Functions, $\Psi^{\circ}$ & Atoms, $N /$ Phases & $\mathrm{O}, 8$ & $\mathrm{~S}, 16$ & Se, 34 & Te, 52 & Po, 84 & Equations \\
\hline \multirow[t]{3}{*}{$\Delta_{\mathrm{f}} G^{\mathrm{o}}$} & gaseous & 231.8 & 197.6 & 166.7 & 154.9 & 106.5 & $\begin{array}{l}\Delta_{\mathrm{f}} G^{\mathrm{o}}=(229.9 \pm 9.3)-(1.5 \pm 0.2) N r 0.976 \\
S_{o} 11.9, n 5^{\text {a }}\end{array}$ \\
\hline & condensed & & 0.4 & 2.6 & & & \\
\hline & gaseous & 249.2 & 278.8 & 206.7 & 194.5 & 144.1 & $\begin{array}{l}\Delta_{\mathrm{f}} H^{\mathrm{o}}=(276.8 \pm 15.8)-(1.6 \pm 0.3) N r 0.941, \\
S_{o} 20.3, n 5^{\mathrm{a}}\end{array}$ \\
\hline$\Delta_{\mathrm{f}} H^{\circ}$ & condensed & & 1.4 & 6.7 & 11.2 & & $\begin{array}{l}\Delta_{\mathrm{H}} H^{\circ}=(-2.8 \pm 0.5)+(0.3 \pm 0.01) N r 0.998, \\
S_{o} 0.3, n 3^{\text {a }}\end{array}$ \\
\hline${ }_{\mathrm{i}} \mathrm{S}^{\mathrm{O}}$ & gaseous & 161.1 & 167.7 & 176.6 & 182.6 & 188.8 & $\begin{array}{l}S^{\mathrm{o}}=(161.6 \pm 2.6)+(0.3 \pm 0.05) N r 0.966, S_{\mathrm{o}} \\
3.3, n 5^{\mathrm{a}}\end{array}$ \\
\hline & condensed & & 35.3 & 48.9 & 49.7 & 64.8 & ${ }_{0}^{0}=(30.9 \pm 3.9)+(0.4 \pm 0.1) N r 0.968, S_{0}$ \\
\hline
\end{tabular}




\begin{tabular}{|c|c|c|c|c|c|c|c|}
\hline \multirow{2}{*}{$C p$} & gaseous & \multirow[t]{2}{*}{21.9} & 23.7 & 21.0 & \multicolumn{2}{|l|}{24.3} & \multirow{2}{*}{$\begin{array}{l}3.7, n 4^{\mathrm{a}} \\
C_{p}=(22.2 \pm 2.6)+(0.04 \pm 0.03) N r 0.806, S_{0} \\
1.0, n 3\left(\text { no Se) }{ }^{\mathrm{a}}\right. \\
C_{p}=(35.7 \pm 4.4)-(0.1 \pm 0.08) N r 0.768, S_{0} \\
4.2, n 3\left(\text { no Te) }{ }^{\mathrm{a}}\right.\end{array}$} \\
\hline & condensed & & 31.7 & 35.6 & 25.7 & 26.4 & \\
\hline \multicolumn{8}{|c|}{ Sixth Lateral Group } \\
\hline Functions, $\Psi^{\circ}$ & Atoms, $N /$ Phases & & $\mathrm{Cr}, 24$ & Mo, 42 & $\mathrm{~W}, 74$ & & Equations \\
\hline \multirow[t]{2}{*}{$\Delta_{\mathrm{f}} G^{\mathrm{o}}$} & gaseous & & 352.6 & 604.9 & 802.3 & & $\begin{array}{l}\Delta_{\mathrm{f}} G^{\mathrm{o}}=(182.2 \pm 103.8)+(8.7 \pm 2.0) N r \\
0.973, S_{o} 72.9, n 3^{\mathrm{a}}\end{array}$ \\
\hline & condensed & & & & 27.8 & & \\
\hline \multirow[t]{2}{*}{$\Delta_{\mathrm{f}} H^{\circ}$} & gaseous & & 397.5 & 660.2 & 844.3 & & $\begin{array}{l}\Delta_{\mathrm{f}} H^{\mathrm{o}}=(234.3 \pm 117.0)+(8.6 \pm 2.3) \mathrm{Nr} \\
0.966, S_{o} 82.1, n 3^{\mathrm{a}}\end{array}$ \\
\hline & $\begin{array}{l}\text { condensed } \\
\text { gaseous }\end{array}$ & & 174.2 & 181.8 & $\begin{array}{l}30.1 \\
173.8\end{array}$ & & No correlation \\
\hline \multirow[t]{2}{*}{${ }_{\mathrm{f}} \mathrm{S}^{\mathrm{O}}$} & condensed & & 23.8 & 28.6 & 32.8 & & $\begin{array}{l}{ }_{\mathrm{A}} S^{\mathrm{o}}=(20.3 \pm 1.8)+(0.2 \pm 0.03) N r 0.980, S_{\mathrm{o}} \\
1.2, n 3^{\mathrm{a}}\end{array}$ \\
\hline & gaseous & & 46.9 & & 21.3 & & \\
\hline$C p$ & condensed & & 23.3 & 23.5 & 24.2 & & $\begin{array}{l}C p=(22.8 \pm 0.1)+(0.02 \pm 0.01) N r 0.989, \\
S_{o} 0.1, n 3^{\text {a }}\end{array}$ \\
\hline \multicolumn{8}{|c|}{ Seventh Main Group } \\
\hline Functions, $\Psi^{\circ}$ & Atoms, $N /$ Phases & $\mathrm{F}, 9$ & $\mathrm{Cl}, 17$ & $\mathrm{Br}, 35$ & I, 53 & At, 85 & Equations \\
\hline$\Delta_{\mathrm{f}} G^{\mathrm{o}}$ & gaseous & 62.4 & 105.3 & 82.4 & 70.2 & 54.4 & $\begin{array}{l}\Delta_{\mathrm{f}} G^{\mathrm{o}}=(112.2 \pm 6.6)-(0.7 \pm 0.1) N r 0.972 \\
S_{o} 6.2, n 4(\text { no F })^{\mathrm{a}}\end{array}$ \\
\hline$\Delta_{\mathrm{f}} H^{\circ}$ & gaseous & 78.9 & 121.1 & 111.8 & 106.8 & 92.0 & $\begin{array}{l}\Delta_{\mathrm{f}} H^{\mathrm{o}}=(127.8 \pm 1.4)-(0.4 \pm 0.03) N r 0.996 \\
S_{o} 1.3, n 4(\text { no F })^{\mathrm{a}}\end{array}$ \\
\hline${ }_{\mathrm{f}} \mathrm{S}^{\mathrm{O}}$ & gaseous & 158.7 & 165.1 & 174.9 & 180.7 & 186.9 & $\begin{array}{l}\mathrm{f} S^{\mathrm{o}}=(158.4 \pm 2.8)+(0.4 \pm 0.1) N r 0.963, S_{\mathrm{o}} \\
3.5, n 5^{\mathrm{a}}\end{array}$ \\
\hline & gaseous & 22.8 & 21.8 & & & & \\
\hline \multicolumn{8}{|c|}{ Seventh Lateral Group } \\
\hline Functions, $\Psi^{\circ}$ & Atoms, $N /$ Phases & & $\mathrm{Mn}, 25$ & Tc, 43 & Re, 75 & & Equations \\
\hline$\Delta_{\mathrm{f}} G^{\mathrm{o}}$ & gaseous & & 238.7 & 604.5 & 733.4 & & $\begin{array}{l}\Delta_{\mathrm{f}} G^{\mathrm{o}}=(86.5 \pm 219.5)+(9.2 \pm 4.2) \mathrm{Nr} \\
0.909, S_{o} 151.3, n 3^{\mathrm{a}}\end{array}$ \\
\hline$\Delta_{\mathrm{f}} H^{\mathrm{o}}$ & gaseous & & 290.0 & 648.5 & 778.6 & & $\begin{array}{l}\Delta_{\mathrm{f}} H^{\mathrm{o}}=(138.2 \pm 213.5)+(9.1 \pm 4.1) N r \\
0.911, S_{o} 147.2, n 3^{\mathrm{a}}\end{array}$ \\
\hline${ }_{f} S^{0}$ & gaseous & & 32.0 & 180.9 & & & \\
\hline $\mathrm{F}$ & condensed & & 32.0 & 49.7 & 36.5 & & No correlation \\
\hline & gaseous & & 27.6 & & & & \\
\hline $\mathrm{Cp}$ & condensed & & 25.5 & 25.8 & 25.7 & & No correlation \\
\hline \multicolumn{8}{|c|}{ First Group of Transitive Elements } \\
\hline Functions, $\Psi^{\circ}$ & Atoms, $N /$ Phases & & $\mathrm{Fe}, 26$ & Co, 27 & $\mathrm{Ni}, 28$ & & Equations \\
\hline$\Delta_{\mathrm{f}} G^{\mathrm{o}}$ & gaseous & & 372.0 & 380.5 & 378.3 & & $\begin{array}{l}\Delta_{\mathrm{f}} G^{\mathrm{o}}=(291.9 \pm 83.3)+(3.1 \pm 3.1) N r \\
0.714, S_{o} 4.4, n 3^{\mathrm{a}}\end{array}$ \\
\hline$\Delta_{\mathrm{f}} H^{\mathrm{o}}$ & gaseous & & 417.7 & 425.1 & 429.6 & & $\begin{array}{l}\Delta_{\mathrm{f}} H^{\mathrm{o}}=(263.5 \pm 23.0)+(5.9 \pm 0.8) N r 0.990, \\
S_{o} 1.2, n 3^{\mathrm{a}}\end{array}$ \\
\hline & gaseous & & 180.4 & 179.4 & 182.1 & & \\
\hline${ }_{\mathrm{f}} S^{\circ}$ & condensed & & 34.3 & 30.0 & 29.9 & & $\begin{array}{l}\mathrm{f} S^{0}=(90.8 \pm 32.7)-(2.2 \pm 1.2) N r 0.876, S_{\mathrm{o}} \\
1.7, n 3^{\mathrm{a}}\end{array}$ \\
\hline $\mathrm{Cp}$ & gaseous & & 25.7 & 23.0 & 23.3 & & $\begin{array}{l}C_{p}=(55.0 \pm 22.6)-(1.1 \pm 0.8) N r 0.808, S_{\circ} \\
1.2, n 3^{a}\end{array}$ \\
\hline & condensed & & 25.0 & 24.8 & 26.1 & & $\begin{array}{l}C_{\mathrm{p}}=(10.4 \pm 12.0)+(0.5 \pm 0.4) N r 0.786, S_{\mathrm{o}} \\
0.6, n 3^{\mathrm{a}}\end{array}$ \\
\hline \multicolumn{8}{|c|}{ Second Group of Transitive Elements } \\
\hline Functions, $\Psi^{\circ}$ & Atoms, $N /$ Phases & & $\mathrm{Ru}, 44$ & $\mathrm{Rh}, 45$ & $\mathrm{Pd}, 46$ & & Equations \\
\hline$\Delta_{\mathrm{f}} G^{\mathrm{o}}$ & gaseous & & 601.4 & 510.9 & 354.8 & & $\begin{array}{l}\Delta_{\mathrm{f}} G^{\mathrm{o}}=(6038 \pm 851)-(123.3 \pm 3.5) N r \\
0.988, S_{o} 26.7, n 3^{\mathrm{a}}\end{array}$ \\
\hline$\Delta_{\mathrm{f}} H^{\mathrm{o}}$ & gaseous & & 648.1 & 556.5 & 393.3 & & $\begin{array}{l}\Delta_{\mathrm{f}} H^{\mathrm{o}}=(6265 \pm 930)-(127.4 \pm 20.7) N r \\
0.987, S_{o} 29.2, n 3^{\mathrm{a}}\end{array}$ \\
\hline & gaseous & & 186.4 & & 166.9 & & \\
\hline \multirow[t]{2}{*}{${ }_{\mathrm{f}} \mathrm{S}^{\mathrm{o}}$} & condensed & & 28.5 & 31.5 & 37.9 & & $\begin{array}{l}{ }_{f} S^{0}=(-178.9 \pm 44.2)+(4.7 \pm 1.0) N r 0.979, \\
S_{0} 1.4, n 3^{\mathrm{a}}\end{array}$ \\
\hline & gaseous & & 21.5 & 21.0 & & & \\
\hline$C p$ & condensed & & 23.8 & 25.0 & 26.0 & & $\begin{array}{l}C_{p}=(-24.6 \pm 2.6)+(1.0 \pm 0.4) N r 0.998, S_{o} \\
0.1, n 3^{\mathrm{a}}\end{array}$ \\
\hline \multicolumn{8}{|c|}{ Third Group of Transitive Elements } \\
\hline Functions, $\Psi^{\circ}$ & Atoms, $N /$ Phases & & Os, 76 & Ir, 77 & $\mathrm{Pt}, 78$ & & Equations \\
\hline$\Delta_{\mathrm{f}} G^{\mathrm{o}}$ & gaseous & & 621.8 & 622.3 & 519.1 & & $\begin{array}{l}\Delta_{\mathrm{f}} G^{\mathrm{o}}=(4541 \pm 2305)-(51.3 \pm 29.9) N r \\
0.864, S_{o} 42.3, n 3^{\mathrm{a}}\end{array}$ \\
\hline$\Delta_{\mathrm{f}} H^{\circ}$ & gaseous & & 790.7 & 673.2 & 564.0 & & $\Delta_{\mathrm{f}} H^{\mathrm{o}}=(9404 \pm 172)-(113.3 \pm 2.2) \mathrm{N} r$ \\
\hline
\end{tabular}




\begin{tabular}{lllll}
\hline & & & & $0.995, S_{o} 3.2, n 3^{\text {a }}$ \\
${ }_{\mathrm{f}} \mathrm{S}^{\mathrm{O}}$ & gaseous & 192.5 & 178.4 & \\
& condensed & & 35.5 & 41.6 \\
$\mathrm{C} p$ & gaseous & & 25.5 & \\
\hline
\end{tabular}

${ }^{\mathrm{a}}$ Data have been used from Ref. [8]; ${ }^{\mathrm{b}}$ Data have been used from Ref. [9].

Table 2. The calculated thermodynamic functions $\left(\mathrm{kJ} \mathrm{mol}^{-1}\right.$ and $\left.\mathrm{J} \mathrm{mol}^{-1} \mathrm{~K}^{-1}\right)$ in the condensed state, which are given as bold type

\begin{tabular}{|c|c|c|c|c|c|c|c|c|c|c|c|c|}
\hline Func-tions & $\begin{array}{l}\text { Atoms, } N \\
\text { /Phases }\end{array}$ & H, 1 & $\mathrm{Ca}, 20$ & C, 6 & $\mathrm{~N}, 7$ & 0,8 & $P, 15$ & $\mathrm{Sb}, 51$ & Bi, 83 & Sn, 50 & $\mathrm{~Pb}, 82$ & Po, 84 \\
\hline \multirow{2}{*}{$\Delta_{\mathrm{f}} G^{\mathrm{o}}$} & gaseous & 103.0 & 142.8 & 669.5 & 455.6 & 231.8 & 292.1 & 222.2 & 168.2 & 267.3 & 162.9 & 106.5 \\
\hline & condensed & 1.0 & 40.3 & 2.9 & & & 12.1 & & & & 2.2 & \\
\hline \multirow{2}{*}{$\Delta_{\mathrm{f}} H^{\mathrm{o}}$} & gaseous & 218.0 & 176.6 & 715.0 & 472.8 & 249.2 & 333.9 & 262.3 & 207.1 & 302.1 & 195.8 & 144.1 \\
\hline & condensed & 2.4 & 7.79 & 16.5 & 316.4 & -0.4 & 18.1 & 10.6 & 74.3 & 2.1 & 4.3 & 22.4 \\
\hline \multirow{2}{*}{${ }_{\mathrm{f}} S^{\mathrm{O}}$} & gaseous & 144.1 & 154.8 & 157.9 & 153.2 & 161.1 & 163.1 & 175.8 & 186.9 & 168.3 & 175.3 & 188.8 \\
\hline & condensed & 37.9 & 45.5 & 5.8 & & & 41.1 & 45.7 & 56.8 & 51.4 & 71.7 & 64.8 \\
\hline \multirow{2}{*}{$C p$} & gaseous & 114.7 & & 20.4 & 32.9 & 21.9 & 20.8 & & 20.8 & 37.3 & 50.1 & \\
\hline & condensed & & 26.3 & 6.1 & & 31.7 & 26.3 & 25.2 & 25.6 & 27.0 & 26.8 & 26.4 \\
\hline
\end{tabular}

Table 3. Thermodynamic functions and calculated through Eq. 1 ( $\pm 1.5 \%)$ the atomizations in the gaseous and condensed phases $\left(\mathrm{kJ} \mathrm{mol}^{-1}\right.$ and $\left.J \mathrm{~mol}^{-1} \mathrm{~K}^{-1}\right)$ of some linear and cyclic alkane

\begin{tabular}{|c|c|c|c|c|c|c|}
\hline \multirow{2}{*}{ No, Compound, formula, $N$} & \multicolumn{4}{|l|}{ Free energy } & \multicolumn{2}{|c|}{ Heat contribution (enthalpy) } \\
\hline & $\Delta_{\mathrm{f}} G^{0}$, gas & $\Delta_{\mathrm{f}} G^{0}$, cond & $\Delta_{\mathrm{a}} G^{\mathrm{ob}}$, gas & $\Delta_{\mathrm{a}} G^{0 \mathrm{~b}}$, cond & $\Delta_{\mathrm{f}} H^{0}$, gas & $\Delta_{\mathrm{f}} H^{0}$, cond \\
\hline \multicolumn{7}{|l|}{ Linear alkanes } \\
\hline I, Methane, $\mathrm{CH}_{4}, 8$ & $-50.6^{[10]}$ & $-31.0^{a} \pm 1.5$ & $1132.1 \pm 17.0$ & $37.9 \pm 0.6$ & $-74.9^{[9]}$ & $-70.8^{c} \pm 0.3$ \\
\hline II, Ethane, $\mathrm{C}_{2} \mathrm{H}_{6}, 14$ & $-33.0^{[10]}$ & $-25.6^{a} \pm 1.3$ & $1990.0 \pm 29.8$ & $37.4 \pm 0.6$ & $-84.7^{[11]}$ & $-96.6^{c} \pm 0.5$ \\
\hline III, Propane, $\mathrm{C}_{3} \mathrm{H}_{8}, 20$ & $-23.0^{[10]}$ & $-20.2^{a} \pm 1.0$ & $2855.5 \pm 43.0$ & $39.7 \pm 0.6$ & $-104.7^{[10]}$ & $-122.4^{c} \pm 0.6$ \\
\hline IV, $n$-Pentane, $\mathrm{C}_{5} \mathrm{H}_{12}, 32$ & $-8.4^{[10]}$ & $-9.5^{[10]}$ & $4591.9 \pm 68.9$ & $36.0 \pm 0.5$ & $-146.8^{[4]} \pm 0.6$ & $-173.5^{[5]} \pm 0.6$ \\
\hline $\mathrm{V}, n$-Oktane, $\mathrm{C}_{8} \mathrm{H}_{18}, 50$ & $16.7^{[4]} \pm 0.9$ & $6.4^{[4]}$ & $7193.3 \pm 107.9$ & $34.8 \pm 0.5$ & $-208.4^{[13]} \pm 0.7$ & $-250.0^{[13]} \pm 0.8$ \\
\hline \multicolumn{7}{|l|}{ Cyclic alkanes } \\
\hline VI, Cyclohexane, $\mathrm{C}_{6} \mathrm{H}_{12}, 36$ & $31.8^{[10]}$ & $26.7^{[10]}$ & $5221.2 \pm 78.3$ & $2.7 \pm 0.04$ & $-123.0^{[10]}$ & $-156.2^{[10]}$ \\
\hline VII, Cyclopentane, $\mathrm{C}_{5} \mathrm{H}_{10}, 30$ & $38.6^{[10]}$ & $36.5^{[3]}$ & $4438.9 \pm 65.1$ & $-12.0 \pm 0.2$ & $-76.9^{[14]}$ & $-105.6^{[14]} \pm 1.8$ \\
\hline
\end{tabular}

Table 3. Continued

\begin{tabular}{|c|c|c|c|c|c|c|}
\hline \multirow{2}{*}{ No, Compound, xxxformula, $N$} & \multicolumn{2}{|c|}{ Heat contribution (enthalpy) } & \multicolumn{4}{|l|}{ Entropy } \\
\hline & $\Delta_{\mathrm{a}} H^{\mathrm{ob}}$, gas & $\Delta_{\mathrm{a}} H^{\mathrm{ob}}$, cond & ${ }_{\mathrm{f}} \mathrm{S}^{0}$, gas & ${ }_{\mathrm{p}} \mathrm{S}^{\mathbf{0}}$, cond & ${ }_{\mathrm{a}} S^{\mathbf{o b}}$, gas & ${ }_{\mathrm{a}} S^{\mathrm{ob}}$, cond \\
\hline \multicolumn{7}{|l|}{ Linear alkanes } \\
\hline I, Methane, $\mathrm{CH}_{4}, 8$ & $1661.9 \pm 25.0$ & $96.9 \pm 1.4$ & $188.7^{[9]}$ & $106.2^{[9]} \pm .61$ & $545.6 \pm 8.2$ & $51.2 \pm 0.8$ \\
\hline II, Ethane, $\mathrm{C}_{2} \mathrm{H}_{6}, 14$ & $2822.5 \pm 42.3$ & $144.0 \pm 2.1$ & $230.0^{[10]}$ & $126.7^{[13]}$ & $950.4 \pm 14.2$ & $112.3 \pm 1.7$ \\
\hline III, Propane, $\mathrm{C}_{3} \mathrm{H}_{8}, 20$ & $3993.7 \pm 60.0$ & $188.5 \pm 2.8$ & $270.0^{[10]}$ & $171.0^{[12]}$ & $1356.5 \pm 20.3$ & $149.6 \pm 2.2$ \\
\hline $\mathrm{IV}, n$-Pentane, $\mathrm{C}_{5} \mathrm{H}_{12}, 32$ & $6337.8 \pm 95.1$ & $284.8 \pm 4.3$ & $347.8^{[4]} \pm 0.8$ & $263.5^{[4]}$ & $2170.9 \pm 32.6$ & $220.3 \pm 3.3$ \\
\hline $\mathrm{V}, n$-Oktane, $\mathrm{C}_{8} \mathrm{H}_{18}, 50$ & $9852.4 \pm 147.8$ & $425.2 \pm 6.4$ & $467.1^{[4]} \pm 0.9$ & $361.2^{[4]}$ & $3389.9 \pm 50.8$ & $367.4 \pm 5.5$ \\
\hline VI, Cyclohexane, $\mathrm{C}_{6} \mathrm{H}_{12}, 36$ & $7029.0 \pm 105.4$ & $284.0 \pm 4.3$ & $298.0^{[10]}$ & $204.0^{[10]}$ & $2378.6 \pm 35.7$ & $285.6 \pm 4.3$ \\
\hline VII, Cyclopentane, $\mathrm{C}_{5} \mathrm{H}_{10}, 30$ & $5831.9 \pm 87.5$ & $212.1 \pm 3.2$ & $293.0^{[10]}$ & $49.0^{[10]}$ & $1937.5 \pm 29.1$ & $359.0 \pm 5.4$ \\
\hline
\end{tabular}

${ }^{\text {a }}$ Calc. via Eq. (3) in Ref. [4]; ${ }^{b}$ Calc. via Eq. (1) with the use of $\Delta_{\mathrm{f}} G^{\mathrm{o}}, \Delta_{\mathrm{f}} H^{\mathrm{o}}, \mathrm{f}_{\mathrm{f}} S^{\mathrm{o}}$ functions; ${ }^{\mathrm{c}}$ Calc. via Eq. (7) in Ref. [4].

Table 4. The calculatedof the gaseous and condensed inner (i) and cyclic (c) bond strength $\left(S_{b} \pm 1.5 \%, \quad \mathrm{~kJ} \mathrm{~mol}^{-1}\right.$ and $J$ mol $\left.{ }^{-1} \mathrm{~K}^{-1}\right)$ on the basis of their thermodynamic functions

\begin{tabular}{|c|c|c|c|c|c|c|}
\hline \multirow{2}{*}{ No, Compound } & \multicolumn{2}{|c|}{$S_{b}$ through free energy, $G^{0}$} & \multicolumn{2}{|c|}{$S_{b}$ through enthalpy, $H^{0}$} & \multicolumn{2}{|c|}{$S_{b}$ through entropy, $S^{0}$} \\
\hline & C-H & $\mathrm{C}-\mathrm{C}$ & C-H & $\mathrm{C}-\mathrm{C}$ & C-H & $\mathrm{C}-\mathrm{C}$ \\
\hline \multicolumn{7}{|l|}{ Linear alkanes } \\
\hline I, Methane & $\begin{array}{l}\text { gas } 283.0 \pm 0.4 \\
\text { cond } 9.5 \pm 0.1\end{array}$ & & $\begin{array}{l}\text { gas } 415.5 \pm 6.2 \\
\text { Lit } 439.3 \pm 0.4^{[2]} \\
\text { cond } 24.2 \pm 0.4\end{array}$ & & $\begin{array}{l}\text { gas } 136.4 \pm 2.0 \\
\text { cond } 12.8 \pm 0.2\end{array}$ & \\
\hline II, Ethane & & $\begin{array}{l}\text { gas } 292.0 \pm 4.4 \\
\text { cond }-19.6 \pm 0.3\end{array}$ & & $\begin{array}{l}\text { gas } 329.5 \pm 4.9 \\
\text { Lit } 377.4 \pm 0.8^{[2]} \\
\text { cond }-1.2 \pm 0.01\end{array}$ & & $\begin{array}{l}\text { gas } 132.0 \pm 2.0 \\
\text { cond } 35.5 \pm 0.5\end{array}$ \\
\hline III, Propane & $\begin{array}{l}\text { gas (i) } 286.7 \pm 4.3 \\
\text { cond (i) } 10.9 \pm 0.2\end{array}$ & & $\begin{array}{l}\text { gas (i) } 420.8 \pm 6.3 \\
\text { Lit (i) } 410.5 \pm 2.9^{[2]} \\
\text { cond (i) } 22.8 \pm 0.3\end{array}$ & & $\begin{array}{l}\text { gas (i) } 137.0 \pm 2.0 \\
\text { cond (i) } 0.9 \pm 0.01\end{array}$ & \\
\hline IV, $n$-Pentane & $\begin{array}{l}\text { gas (i) } 287.6 \pm 4.3 \\
\text { cond (i) } 9.5 \pm 0.08\end{array}$ & $\begin{array}{l}\text { gas (i) } 294.8 \pm 4.4 \\
\text { cond (i) }-23.6 \pm 0.3\end{array}$ & $\begin{array}{l}\text { gas (i) } 421.1 \pm 6.3 \\
\text { Lit (i) } 415.1^{[2]}\end{array}$ & $\begin{array}{l}\text { gas (i) } 330.5 \pm 5.0 \\
\text { Lit (i) } 365.3 \pm 2.9^{[2]}\end{array}$ & $\begin{array}{l}\text { gas (i) } 137.4 \pm 2.1 \\
\text { cond (i) } 0.3 \pm 0.005\end{array}$ & $\begin{array}{l}\text { gas (i) } 133.2 \pm 2.0 \\
\text { cond (i) } 33.5 \pm 0.5\end{array}$ \\
\hline
\end{tabular}




\begin{tabular}{|c|c|c|c|c|c|c|}
\hline \multirow{2}{*}{ No, Compound } & \multicolumn{2}{|c|}{$S_{b}$ through free energy, $G^{0}$} & \multicolumn{2}{|c|}{$S_{b}$ through enthalpy, $H^{0}$} & \multicolumn{2}{|c|}{$S_{b}$ through entropy, $S^{0}$} \\
\hline & C-H & $\mathrm{C}-\mathrm{C}$ & C-H & $\mathrm{C}-\mathrm{C}$ & C-H & $\mathrm{C}-\mathrm{C}$ \\
\hline $\mathrm{V}, n$-Oktane & $\begin{array}{l}\text { gas (i) } 287.6 \pm 4.3 \\
\text { cond (i) } 9.6 \pm 0.1\end{array}$ & $\begin{array}{l}\text { gas (i) } 294.2 \pm 4.4 \\
\text { cond (i) }-22.8 \pm 0.3\end{array}$ & $\begin{array}{l}\text { cond (i) } 24.1 \pm 0.4 \\
\text { gas (i) } 421.1 \pm 6.3 \\
\text { Lit (i) } 415.1^{[2]} \\
\text { cond (i) } 24.0 \pm 0.4\end{array}$ & $\begin{array}{l}\text { cond (i) } 2.6 \pm 0.03 \\
\text { gas (i) } 330.2 \pm 4.9 \\
\text { Lit (i) } 364.0 \pm 3.8^{[2]} \\
\text { cond (i) } 1.8 \pm 0.02\end{array}$ & $\begin{array}{l}\text { gas (i) } 137.3 \pm 2.0 \\
\text { cond (i) } 3.5 \pm 0.05\end{array}$ & $\begin{array}{l}\text { gas (i) } 132.7 \pm 2.0 \\
\text { cond (i) } 41.8 \pm 0.6\end{array}$ \\
\hline $\begin{array}{l}\text { The middle values } \\
\text { of } S_{b} \text { of C-H and } \\
\text { C-C bonds } \\
\text { Cycloalkanes }\end{array}$ & $\begin{array}{l}\text { gas } 286.2 \pm 4.3 \\
\text { cond } 9.9 \pm 0.1\end{array}$ & $\begin{array}{l}\text { gas } 293.7 \pm 4.4 \\
\text { cond }-22.0 \pm 0.3\end{array}$ & $\begin{array}{l}\text { gas } 419.6 \pm 6.3 \\
\text { cond } 23.8 \pm 0.3\end{array}$ & $\begin{array}{l}\text { gas } 330.1 \pm 4.9 \\
\text { cond } 1.1 \pm 0.01\end{array}$ & $\begin{array}{l}\text { gas } 137.0 \pm 2.0 \\
\text { cond: hard define }\end{array}$ & $\begin{array}{l}\text { gas } 132.6 \pm 2.0 \\
\text { cond } 36.9 \pm 0.5\end{array}$ \\
\hline VI, Cyclohexane & & $\begin{array}{l}\text { gas (c) } 296.8 \pm 4.3 \\
\text { cond (c) }-21.3 \pm 0.3\end{array}$ & Lit (e) $403.3 \pm 2.5^{[2]}$ & $\begin{array}{l}\text { gas (c) } 329.9 \pm 4.9 \\
\text { Lit (c) } 371.5 \pm 2.9^{[2]} \\
\text { cond (c) } 1.7 \pm 0.02\end{array}$ & & $\begin{array}{l}\text { gas (c) } 123.6 \pm 1.8 \\
\text { cond (c) } 22.0 \pm 0.3\end{array}$ \\
\hline VII, Cyclopentane & & $\begin{array}{l}\text { gas (c) } 314.4 \pm 4.7 \\
\text { cond (c) }-24.2 \pm 0.4\end{array}$ & Lit (e) $400.0 \pm 4.2^{[2]}$ & $\begin{array}{l}\text { gas (c) } 324.8 \pm 4.9 \\
\text { Lit (c) } 371.5 \pm 2.9^{[2]} \\
\text { cond (c) }-3.2 \pm 0.04\end{array}$ & . & $\begin{array}{l}\text { gas (c) } 113.5 \pm 1.7 \\
\text { cond (c) } 71.6 \pm 1.1\end{array}$ \\
\hline
\end{tabular}

\section{Conclusions}

On the base of the consideration of all presented data is possible to make some conclusions.

First of all it was established that practically all thermodynamic functions and heat capacities of simple atoms of Mendeleev's table are depended from the number of electrons in they.

The second of them is connected with the necessity to present all thermodynamic functions of the calculated bonds in organic or organometallic compounds, that to have a really understanding about the bond strength of they.

The third is connected with the necessity of calculations of all bonds in the condensed phase especially because the large number of heteroatomic compounds have a reactivity in the condensed conditions than in gaseous phase.

\section{References}

[1] Ovchinnikov V.V., Lapteva L.I., Makeeva T.B., Kudryavtzev V.Yu., Sagadeev E.V. and Konovalov A.I., Enthalpies of formation and bond energies of P(III) and As(III) compounds. Phosphorus, Sulfur, Silicon and the Related Elements, 109, 97-100 (1996).

[2] Yu-Ran Luo, Handbook of Bond Dissociation Energies in Organic Compounds, CRC PRESS, Boca Ranton-LondonNew York-Washington, D.C., 362 pp. (2003).

[3] Ovchinnikov V.V., Thermochemistry of heteroatomic compounds: analysis and calculation of thermodynamic functions of organometallic compounds of I-IV groups of Mendeleev's Periodic table, AJPC, 2, 52-59 (2013).

[4] Ovchinnikov V.V., Thermochemistry of Heteroatomic Compounds: Analysis and Calculation of Some Thermodynamic Functions of Saturated Alkanes, ACSJ, 4(1), $1-13$ (2014).
[5] Ovchinnikov V.V., Thermochemistry of Heteroatomic Compound: Some thermodynamic Aspects of Combustion and Formation of Carbohydrates of Different Structures, Chem. Journal. 2, 2, 59-65 (2013).

[6] Ovchinnikov V.V., Thermochemistry of heteroatomic compounds: analysis and calculation of thermodynamic functions of amino acids and some peptides of different space structure, AJPC, 1, 1, 8-15 (2013).

[7] Ovchinnikov V.V., Thermochemistry of Heteroatomic Compounds: Enthalpy of Combustion of Organic Compounds of Group I-VII Elements, Doklady Physical Chemistry, 443, 49-52 (2012).

[8] Karapetyanz M.Kh. and Karapetyanz M.L., The basic thermodynamic constants of inorganic and organic chemistry, Moscow, Chemistry, 471 pp. (1968).

[9] Chase, M.W., Jr., NIST-JANAF Themochemical Tables, Fourth Edition, J. Phys. Chem. Ref. Data, Monograph 9, 1951 pp. (1998).

[10] Pittam D.A. and Pilcher G., Measurements of heats of combustion by flame calorimetry. Part 8 . Methane, ethane, propane, n-butane and 2-methylpropane, J. Chem. Soc. Faraday Trans. 1, 68, 2224-2229 (1972).

[11] Prosen E.J. and Rossini F.D. Heats of combustion and formation of the paraffin hydrocarbons at $25^{\circ} \mathrm{C}$, J. Res. NBS, 263-267 (1945).

[12] Kemp J.D. and Egan C.J., Hindered rotation of the methyl groups in propane. The heat capacity, vapor pressure, heats of fusion and vaporization of propane. Entropy and density of the gas, J. Am. Chem. Soc., 60, 1521-1525 (1938).

[13] Witt R.K. and Kemp J.D., The heat capacity of ethane from $15^{\circ} \mathrm{K}$ to the boiling point. The heat of fusion and the heat of vaporization, J. Am. Chem. Soc., 59, 273-276 (1937).

[14] Spitzer R. and Huffman H.M., The heats of combustion of cyclopentane, cyclohexane, cycloheptane and cyclooctane. J. Am. Chem. Soc., 69, 211-213 (1947). 